\title{
Abkürzungen und Schrifttum
}

Abs.

Absatz

ÄndVO

Änderungsverordnung

a. F. alte Fassung

AHK Ges. Nr. 8 . .... Gesetz Nr. 8 der Alliierten Hohen Kommission: Gewerbliche, literarische und künstlerische Eigentumsrechte ausländischer Staaten und Staatsangehöriger vom 20. 10. 1949

Allfeld

Allfeld, Kommentar zum Patent-, Geschmacks- und Gebrauchsmustergesetz 1904

a. M. anderer Meinung

Anm. Anmerkung

AnnStG Gesetz über die Errichtung von Annahmestellen für Patent-, Gebrauchsmuster- und Warenzeichenanmeldungen vom 5. 7. 1948

ArbErfG ........... Gesetz über Arbeitnehmererfindungen vom 25. 7. 1957

ArbGG Arbeitsgerichtsgesetz vom 3. 9. 1953

ArmPAnwGebG Gesetz über die Erstattung von Gebühren für im Armenrecht beigeordnete Vertreter in Patent- und Gebrauchsmustersachen vom 18. 7. 1953

AusstellG

Gesetz betreffend den Schutz von Erfindungen, Mustern und Warenzeichen auf Ausstellungen vom 18. 3. 1904

BA bzw. BS . . . . . . . Beschwerdeabteilung des RPA; seit 1936 Beschwerdesenat des RPA bzw. DPA, seit 1961 des Patentgerichts

BAnz. Bundesanzeiger

Baumbach-Lauterbach .. Baumbach-Lauterbach, Zivilprozeßordnung. Komm., C. H. Beck Verlb. München, 27. Aufl. 1963

BB

Der Betriebsberater, Zehntagedienst Verlagsges. Recht und Wirtschaft m. b. H., Heidelberg

Begd. Gesetzesbegründung

Benkard Benkard, Patentgesetz, Gebrauchsmusterschutz, Patentanwaltsgesetz, Komm., C. H. Beck Verlb., München, 4. Aufl. (Bock-Löscher-Ballhaus) 1963

Bernhardt

Bernhardt, Lehrbuch des deutschen Patentrechts, C. H. Beck Verlb., München, 2. Aufl. 1963 


\section{Abkürzungen und Schrifttum}

\begin{tabular}{|c|c|}
\hline BerVO & $\begin{array}{l}\text { Verordnung über das Berufungsverfahren beim Reichs- } \\
\text { gericht in Patentsachen vom 30.9. } 1936\end{array}$ \\
\hline BGB & Bürgerliches Gesetzbuch \\
\hline BGBl. & Bundesgesetzblatt \\
\hline BGH & Bundesgerichtshof \\
\hline BGHZ & $\begin{array}{l}\text { Entscheidungen des Bundesgerichtshofs in Zivilsachen } \\
\text { (Band) }\end{array}$ \\
\hline $\mathrm{Bkm}$. & Bekanntmachung \\
\hline Bl... & $\begin{array}{l}\text { Blatt für Patent-, Muster- und Zeichenwesen mit Ur- } \\
\text { heberrechtsteil. Herausgegeben vom Deutschen Patent- } \\
\text { amt, Carl Heymanns Verl. KG., München }\end{array}$ \\
\hline BMJ & Bundesminister der Justiz \\
\hline BPatGerE & Entscheidungen des Bundespatentgerichts \\
\hline BPG & Bundespatentgericht \\
\hline BRAO & Bundesrechtsanwaltsordnung vom 1. 8. 1959 \\
\hline BS & Beschwerdesenat \\
\hline Busse WZG & $\begin{array}{l}\text { Busse, Warenzeichengesetz nebst Pariser Verbands- } \\
\text { übereinkunft und Madrider Abkommen, Komm., Slg. } \\
\text { Guttentag Nr. 246 Verl. Walter de Gruyter \& Co., } \\
\text { Berlin. 3. Aufl. 1960, Erg. } 1962\end{array}$ \\
\hline
\end{tabular}

Bussmann-Pietzcker ... Bussmann-Pietzcker-Kleine, Gewerblicher Rechtsschutz und Urheberrecht. Verl. Walter de Gruyter \& $\mathrm{Co}$, 3. Aufl. 1962

BVerwG .......... Bundesverwaltungsgericht

Damme-Lutter ....... Damme und Lutter, Das deutsche Patentrecht. Handbuch, Verl. Liebmann, Berlin, 3. Aufl. 1925

DJZ ............ Deutsche Juristenzeitung, C. H. Beck Verlb. Berlin

DPA ............ Deutsches Patentamt, München

DPAVO ........... Verordnung über das Deutsche Patentamt v. 9. 5. 1961

DRiG ............ Deutsches Richtergesetz vom 8. 9. 1961

DRiZ $\ldots \ldots \ldots \ldots$ Deutsche Richterzeitung, Monatsschrift, Carl Heymanns Verl. KG, Köln-Berlin

DVO ........... Durchführungsverordnung

Ebermayer . ....... Ebermayer-Lobe-Rosenberg, Strafgesetzbuch, Komm. (Leipziger Komm.), 8. Aufl. 1957

EGH Ehrengerichtshof für Patentanwälte

Elster

Elster, Urheber- und Erfinder-, Warenzeichen- und Wettbewerbsrecht. Verl. Walter de Gruyter \& Co., Berlin, 2. Aufl. 1928 
Abkürzungen und Schrifttum

Eyermann-Fröhler ..... Eyermann-Fröhler, Verwaltungsgerichtsordnung, Komm., C. H. Beck Verlb., 3. Aufl. 1962

Festschr. 1927 . . . . . . Festschrift des RPA, 1877-1927, herausgeg. vom RPA. Verl. Heymann, Berlin 1927

Festschr. 1952 ....... Festschrift des DPA, Bl. 1952, Juliheft

FGG ............... Gesetz über die Angelegenheiten der freiwilligen Gerichtsbarkeit v. 17. 5. 1898 i. d. F. v. 18. 5. 1957

Furler .......... Furler, Geschmacksmustergesetz, Komm., Carl Heymanns Verl. KG, 2. Aufl. 1956

GbmG ........... Gebrauchsmustergesetz in der Fassung vom 9. 5. 1961

GebG ............ Gesetz über die Gebühren des Patentamts und Patentgerichts i.d. F. vom 9. 5. 1961

Geschäftstät. ....... Geschäftstätigkeit des Reichspatentamts, Ergänzungsband zum Bl.f. PMZ 1901

GewO $\ldots \ldots \ldots \ldots \ldots$ Gewerbeordnung für das Deutsche Reich

GG $\ldots \ldots \ldots \ldots \ldots$ Grundgesetz für die Bundesrepublik Deutschland (Bonner Grundgesetz) vom 23. 5. 1949

GKG ............. Gerichtskostengesetz i. d. F. vom 26. 7. 1957

GrS ............ Großer Senat

GRUR ............ Gewerblicher Rechtsschutz und Urheberrecht. Zeitschrift der deutschen Vereinigung für gewerblichen Rechtsschutz und Urheberrecht, Verl. Chemie $\mathrm{GmbH}$, Weinheim/Bergstr.; hierzu Auslands- und internationaler Teil

GschmG .......... Gesetz betreffend das Urheberrecht an Mustern und Modellen (Geschmacksmustergesetz) vom 11. 1. 1876

GVG ............ Gerichtsverfassungsgesetz

GWB $\ldots \ldots \ldots \ldots \ldots$ Gesetz gegen Wettbewerbsbeschränkungen vom 27. 7. 1957 (Kartellgesetz)

HGB ............. Handelsgesetzbuch

Hubmann ........ Hubmann, Gewerblicher Rechtsschutz, Studienbuch, C. H. Beck Verlb., München 1962

i. d. F. in der Fassung

JMBI. .......... Justizministerialblatt

Isay $\ldots \ldots \ldots \ldots \ldots$ Isay, Patentgesetz und Gesetz betr. den Schutz von Gebrauchsmustern. Komm., Verl. Franz Vahlen, Berlin, 6. Aufl. 1932

JurR ........... Juristische Rundschau, Verl. Walter de Gruyter \& Co., Berlin

JVBl. $\quad \ldots \ldots \ldots \ldots \ldots$ Justizverwaltungsblatt, Heymann Verl.

JW $\ldots \ldots \ldots \ldots \ldots$ Juristische Wochenschrift, Berlin

JZ ............. Juristenzeitung, Verl. J. C. B. Mohr, Tübingen

XVI 


\section{Abkürzungen und Schrifttum}

KG Kammergericht

Kisch Kisch, Handbuch des deutschen Patentrechts. Verl. Bensheimer, Mannheim 1923

Klauer-Möhring ...... Klauer-Möhring, Patentgesetz und Gebrauchsmustergesetz. Komm., Verl. Franz Vahlen, Berlin, 2. Aufl. 1940

Klauer PAnwG ...... Klauer, Patentanwaltsgesetz, Komm., Verl. Vahlen 1933

KO Konkursordnung

Kohler Hb. ......... Kohler, Handbuch des deutschen Patentrechts. Verl. Bensheimer, Mannheim 1900-1901

Kohler Lb. . ......... Kohler, Lehrbuch des Patentrechts, 1909

Krauße ........... Krauße-Katluhn-Lindenmaier, Patentgesetz Komm., Carl Heymanns Verl., Berlin, 3. Aufl. 1944, s. auch Lindenmaier

Kühnemann ......... Kühnemann, Patentgesetz in Pfundtner-Neubert, Das neue Deutsche Reichsrecht. Industrieverl. Späth \& Linde, Berlin 1936

Lauterbach . ........ Lauterbach, Kostengesetze, 14. Aufl. 1960, Nachtrag 1962

Lindenmaier $\ldots \ldots \ldots$ Lindenmaier-Krauße-Katluhn, Patentgesetz, Komm., Carl Heymann Verl. KG, Köln-Berlin, 4. Aufl. 1955/8, Nachtrag 1960

LG Landgericht

LMG $\quad \ldots \ldots \ldots \ldots \ldots$ Lebensmittelgesetz v. 17. 1. 1936, geändert 21. 12. 1958

Lüdecke-Fischer . . . . . L Lüdecke-Fischer, Lizenzverträge, 1957

Lutter ........... Lutter, Patentgesetz mit Gebrauchsmustergesetz, Komm., Verl. Walter de Gruyter \& Co., Berlin, 10. Aufl. 1936

MA ........... Der Markenartikel, Monatsschrift, C. H. Beck Verl., München

MaßnVO ........... Verordnung über Maßnahmen auf dem Gebiet des Patent- und Warenzeichenrechts vom 1. 8. 1953

MDR ........... Monatsschrift für Deutsches Recht, Deutsche Rechtsprechung. Verlags-G.m.b.H., Hamburg

Mitt. ............. Mitteilungen der deutschen Patentanwälte; herausgeg. vom Vorstand der Patentanwaltskammer. Heymanns Verl.

Mittbl. .......... Mitteilungsblatt der Deutschen Vereinigung für gewerblichen Rechtsschutz und Urheberrecht. Verl. J. P. Bachem, Köln

MuW .......... Markenschutz und Wettbewerb, Monatsschrift. Verl. für Staatswissenschaften und Geschichte G.m.b.H., Berlin 
Abkürzungen und Schrifttum

Na bzw. NS . . . . . . . Nichtigkeitsabteilung, seit 1936 Nichtigkeitssenat des RPA bzw. DPA, seit 1961 des Patentgerichts

NJW ............ Neue Juristische Wochenschrift. C. H. Beck Verlb., München

OLG $\ldots \ldots \ldots \ldots \ldots$ Oberlandesgericht

Osterrieth $\ldots \ldots \ldots \ldots$ Osterrieth, Lehrbuch des gewerblichen Rechtsschutzes. Verl. Deichert. 1908

PAnw. . . . . . . . Patentanwalt

PAnwG .......... Patentanwaltsgesetz v. 28. 9. 1933, geändert 2. 7. 1949

PatG bzw. PG ......... Patentgesetz in der Fassung vom 9. 5.1961

PatGebG .......... Gesetz über die Gebühren des Patentamts und Patentgerichts i. d. F. vom 9. 5. 1961

PatVerlG . . ......... Gesetz über die Verlängerung der Dauer bestimmter Patente vom 15. 7. 1951

Pietzcker . . . . . . . . Pietzcker, Patentgesetz, Komm. ( $\$ 1-12$ PatG). Verl. de Gruyter \& Co., Berlin 1929

Pinzger $\quad . . \ldots \ldots \ldots$ Pinzger, Das deutsche Geschmacksmusterrecht, Komm., Verl. Stilke, Berlin 1932

Präsbesch. ......... Präsidialbescheid des RPA bzw. DPA

Propr. ind. ......... La Propriété industrielle, Zeitschrift des internat. Büros für gewerblichen Rechtschutz, Genf

PVU ............. Pariser Verbandsübereinkunft zum Schutz des gewerblichen Eigentums vom 20. 3. 1883, revidiert in Brüssel am 14. 12. 1900; in Washington am 2. 6. 1911; im Haag am 6. 11. 1925, in London am 2. 6. 1934 und in Lissabon am 31. 10. 1958

RAGebO .......... Bundesgebührenordnung für Rechtsanwälte vom 26.7. 1957

Reimer ........... Reimer, Patentgesetz und Gebrauchsmustergesetz, Komm., Carl Heymanns Verl. KG, München. 2. Aufl. 1958

Reimer-Schade $\ldots \ldots \ldots$ Reimer-Schade-Schippel, Das Recht der Arbeitnehmererfindung. Erich Schmidt-Verl., Berlin, 4. Aufl. 1964

RG

Reichsgericht

RGBl ........... Reichsgesetzblatt

RGR Komm. ....... Das Bürgerliche Gesetzbuch. Kommentar der Reichsgerichtsräte. Verl. Walter de Gruyter \& Co., Berlin, 11. Aufl. 1959 ff.

RGSt .......... Entscheidungen des Reichsgerichts in Strafsachen

RGZ ............ Entscheidung des Reichsgerichts in Zivilsachen

RPA $\ldots \ldots \ldots \ldots \ldots$ Reichspatentamt

XVIII 
Abkürzungen und Schrifttum

SaarEinglG . . . . . . . Gesetz über die Eingliederung des. Saarlandes auf dém Gebiet des gewerblichen Rechtsschutzes v. 30.6. 1959

SaatgG Saatgutgesetz vom 27. 6. 1953

Schlegelberger HGB .... Schlegelberger, Handelsgesetzbuch, bearbeitet von Geßler, Hefermehl, Hildebrandt, Schröder. Komm., Verl. für Rechtswissenschaft vorm. Franz Vahlen GmbH, Berlin, 4. Aufl. 1960/62

Seligsohn .......... Seligsohn, Patentgesetz u. Gesetz betr. den Schutz von Gebrauchsmustern. Komm., Verl. Walter de Gruyter \& Co., Berlin, 7. Aufl. 1932

Staub ........... Staub, Kommentar zum Handelsgesetzbuch. Verl. Walter de Gruyter \& Co., Berlin, 14. Aufl. 1933

Stein-Jonas $\ldots \ldots \ldots \ldots$ Stein-Jonas-Schönke-Pohle, Zivilprozeßordnung. Komm., Verl. Mohr, Tübingen, 18. Aufl. 1953-1956

StGB Strafgesetzbuch

StPO ............. Strafprozeßordnung

Taschenbuch $\quad \ldots \ldots$... Taschenbuch des Gewerblichen Rechtsschutzes, herausgegeben v. Deutschen Patentamt, Carl Heymanns Verl. KG, 1955

Tetzner $\ldots \ldots \ldots \ldots$ Tetzner, Patentgesetz. Komm., Fachverlag Dr. N. Stoytscheff, Nürnberg, 2. Aufl. 1951

Trüstedt PA ... .... Trüstedt, Patente und ihre Anmeldung: Leitfaden, Carl Heymanns Verl. KG, München 1957

Trüstedt GA

Trüstedt, Gebrauchsmuster und ihre Anmeldung. Leitfaden, Carl Heymanns Verl. KG, München 1957

1. UG

Erstes Gesetz zur Änderung und Uberleitung von Vorschriften auf dem Gebiet des gewerblichen Rechtsschutzes vom 8. 7. 1949

2. UG

Zweites Gesetz zur Änderung und Ǔberleitung von Vorschriften auf dem Gebiet des gewerblichen Rechtsschutzes vom 2. 7. 1949

3. UG ............. Drittes Gesetz zur Änderung und Úberleitung von Vorschriften auf dem Gebiet des gewerblichen Rechtsschutzes vom 3. 10. 1951

4. UG ........... Viertes Gesetz zur Änderung und Uberleitung von Vorschriften auf dem Gebiet des gewerblichen Rechtsschutzes vom 20. 12. 1951

5. ÜG

Fünftes Gesetz zur Änderung und Überleitung von Vorschriften auf dem Gebiet des gewerblichen Rechtsschutzes vom 18. 7. 1953

6. UG

Sechstes Gesetz zur Änderung und Überleitung von Vorschriften auf dem Gebiet des gewerblichen Rechtsschutzes vom 23. 3. 1961

Ulmer

Ulmer, Urheber- und Verlagsrecht. Springer Verl., Berlin-Göttingen, 2. Aufl. 1960 
Abkürzungen und Schrifttum

UWG Gesetz gegen den unlauteren Wettbewerb v. 7. 6. 1909

VO Verordnung

Völp Völp, Patentgerichtsverfahren, 1961

Voigtländer-ElsterVoigtländer-Elster-Kleine, Urheberrecht, Komm., Verl. Kleine Walter de Gruyter \& Co., Berlin, 4. Aufl. 1952

VwZG Verwaltungszustellungsgesetz vom 3. 7. 1952

AllgVV z. VwZG Allgemeine Verwaltungsvorschriften zum Verwaltungszustellungsgesetz vom 3. 10. 1952

Weber Weber, Die Grenzen des Gebrauchsmusterrechts, Carl Heymanns Verl., Berlin 1934

Wieczorek . . . . . . Wieczorek, Zivilprozeßordnung, Komm., 1957/1959

WRP Wettbewerb in Recht und Praxis. Monatl. Zeitschrift

WuW Wirtschaft und Wettbewerb. Monatl. Zeitschrift

WZG Warenzeichengesetz in der Fassung vom 9. 5. 1961

Zeller Zeller, Gebrauchsmusterrecht. Carl Heymanns Verl. KG, Köln, 2. Aufl. 1952

Zeller, Erfvertragsr. .... Zeller, Erfindervertragsrecht. Carl Heymanns Verl. KG, Köln, 1952

ZfI

Zeitschrift für Industrierecht

ZPO

Zivilprozeßordnung vom 8. 11. 1933, Fassung vom 12. 9.1950 